REVIEWS

\title{
Acinetobacter baumannii biofilm resistance mechanisms and prevention and control of progress
}

\author{
Liying Wang, Lingfeng Wang* \\ The Third Affiliated Hospital of Inner Mongolia Medical University, Baotou, China
}

Received: April 25, 2016

DOI: $10.14725 /$ dcc.v3n2p22
Accepted: May 16, $2016 \quad$ Online Published: June 10, 2016

URL: http://dx.doi.org/10.14725/dcc.v3n2p22

\begin{abstract}
At present, the multiple drug resistance of Acinetobacter baumannii outbreaks worldwide and has intensified the trend, especially in the intensive care unit and burn ward. Generic drug resistant Acinetobacter baumannii is known as the $21^{\text {st }}$ century gram-negative bacterium "MRSA", "Superbugs". In recent years, researches have shown that this is associated with pathogenic bacteria to form biofilms. In this paper, the status of Acinetobacter baumannii infection, biofilm formation, resistance mechanism and prevention in recent years were summarized.
\end{abstract}

Key Words: Acinetobacter baumannii, Biofilms, Resistance mechanisms

Acinetobacter baumannii is a non-fermentable Gramnegative bacillus commonly found in nature and the human body, and is one of the most common opportunistic pathogens in nosocomial infections. Acinetobacter baumannii has been increasing in clinical isolates in recent years, reaching as high as $90 \%$ in burn wards, and multidrug resistant strains are increasing. ${ }^{[1]}$ The rate of resistance to commonly used antibiotics reached $84.96 \% .{ }^{[2]}$ The mortality rate after Acinetobacter baumannii infection increased year by year and the mortality rate in ICU patients was $10 \%$ 43\%. ${ }^{[3]}$ Experts from the US Centers for Disease Control and Prevention estimate that $65 \%$ of human infections are associated with bacterial biofilm (BBF) infection. $42 \%$ of the strains have been clinically detected to form biofilms, of which $73 \%$ Acinetobacter baumannii shows multidrug resistance. ${ }^{[4]}$

\section{Acinetobacter baumannii biofilm forma- tion and drug resistance mechanism}

In order to adapt to the living environment, bacteria are adsorbed on the surface of inert or active materials to form
BBF. ${ }^{[5]}$ Biofilm is the growth mode of bacteria and planktonic state, and its biological characteristics are significantly different from those of planktonic bacteria. First of all, real formation and resistance mechanism of BBF with structural, coordination and function, can protect the membrane of bacteria in the antibacterial which makes it difficult for drugs to play a role, resulting in chronic infection or recurrent, difficult to cure, or even develop into sepsis. ${ }^{[6]}$ The formation of $\mathrm{BBF}$ is a dynamic process in which bacteria first adhere to the surface of human tissues or objects and then exchange information with each other through the acylation of serine lactones, ${ }^{[7]}$ leading to the accumulation of bacteria of the same type. At the same time, extracellular polysaccharide (EPS) form micro-colonies, and multiple microcolonies fuse with each other to form mature biofilms with liquid channels connected to each other. Some of the mature biofilms shed or release planktonic bacteria. These planktonic bacteria continue to adhere, mature and form a biofilm in a suitable environment. Feng et al. ${ }^{[8]}$ used an automatic flow cell system and micro fluidic channel connected to an imaging system, and clearly observed the cycling of Acinetobacter baumannii biofilm on glass. Repeated cycle of the

\footnotetext{
*Correspondence: Lingfeng Wang; E-mail: wlf7413@ vip.sina.com; Address: Department of Burn, The Third Affiliated Hospital of Inner Mongolia Medical University, Baotou, China.
} 
above stage resulted in repeated infections.

\subsection{Expression of unique gene of bacteria in biofilm}

Acinetobacter baumannii biofilm bacteria can express more than $50 \%$ of specific protein products different from planktonic bacteria, resulting in a unique biofilm phenotype. The biofilm formation of Acinetobacter baumannii is related to the up-regulation of genes such as metal ions, plasmids, transposons and integrons, and the expression of outer membrane proteins. ${ }^{[9]}$ It has been reported that in biofilm closed systems, biological signal induced gene expression levels can be increased by 20 times. The expression of intI1 mRNA increases in Acinetobacter baumannii under the biofilm state. Class 1 integron and 16S rRNA methylase genes are together to promote gene movement and spread, so that bacteria highly express biofilm-related gene sequence. The gene csuC, csuD, csuE, ompA, blaPER-1, abaI, ${ }^{[10]}$ six genes of pilus synthetic system, ${ }^{[11]}$ new genotypic ST25 and ST78 ${ }^{[12]}$ play an important role in the adhesion and formation of the biofilm of Acinetobacter baumannii. Xiang $\mathbf{J}$ et al. ${ }^{[13]}$ found that the formation of the biofilm and the increase in the thickness of the clinical strain of Acinetobacter baumannii may be associated with the increase of pgaB transcriptional level. Moreover, the expression of quorum sensing gene AbaI may enhance the expression of pgaB gene, resulting in the formation of extracellular matrix and biofilm and resistance development of Acinetobacter baumannii. However, some scholars believe that AbaI gene is not the only determinant of Acinetobacter baumannii biofilm formation though it is widely present in clinical strains of Acinetobacter baumannii. ${ }^{14]}$ The expression of membrane protein Bap ${ }^{[15]}$ mainly maintains the integrity of the outer membrane. T2 protein family of ribonuclease can promote Acinetobacter baumannii adhesion and motility, and promote the formation of biofilm. ${ }^{[16]}$ It is thought that there is no significant correlation between the biofilm forming ability of Acinetobacter baumannii and genotype. Biofilm formation may be regulated by other genetic factors or environmental factors. ${ }^{[17]}$ At present, there is no final conclusion regarding Acinetobacter baumannii biofilm gene mechanism of resistance and its relationship with the gene, which needs to be further studied.

\subsection{Permeability barrier role of biofilm}

EPS plays an important role in the formation and pathogenesis of biofilm. Its main components are alginate and antibiotic hydrolytic enzymes immobilized on biofilms, which prevent the entry of antibacterial agents into the target and reduce the antibacterial activity. ${ }^{[18]}$ Most of EPS is negatively charged, which absorbs the positively charged amino side chains in polypeptide chains, hinders the penetration of hydrophilic antibiotics into the cell bodies, and significantly reduces the bactericidal ability. This is also the reason why bacteria are not easily removed after biofilm formation. The multi-resistant strain of Acinetobacter baumannii owns $\mathrm{O}$ glycosylation system and capsule synthesis. It is essential to resist antibiotics in the formation of biofilms. If PglC is induced, the initial glycosyltransferase prevents the synthesis of glycoproteins and capsules. The abnormal biofilm structure and virulence of mice can further reduce the genetic and metabolic burden of bacterial cells and enhance the activity of bacteria by sharing polysaccharides. ${ }^{[19,20]}$

\subsection{Change of living environment in biofilm}

The typical characteristic of the biofilm is the micro step gradient of the nutrient concentration, which is closely related to its drug resistance. Many studies have shown that the existence of biofilm and lack of nutrition and oxygen in the membrane of Acinetobacter baumannii result in different bacterial growth rate inside and outside the membrane.

In the use of antibiotics for treatment, bacteria out of biofilm with rapid growth are most sensitive and first killed. The sensitivity of bacteria with slow growth in the membrane decreases and enters into the "hibernation" state. ${ }^{[21]}$ Until the drug stops functioning, residual bacteria quickly multiplies to form a new biofilm by use of dead ones as a nutrient source, which stimulates the recurrence of infection. In addition, there is also significant different in $\mathrm{pH}$ value between inside and outside of biofilm, mainly due to the accumulation of acidic metabolites to reduce drug sensitivity. Changes in osmotic pressure also make a difference in the proportion of bacterial outer membrane proteins, resulting in a decrease in the permeability of biofilms to antibiotics. ${ }^{[22]}$ These factors may be important reasons of multidrug resistance caused by Acinetobacter baumannii.

\subsection{Biofilm sensing}

Quorum sensing (QS) refers to a series of behaviors in which bacteria exchange, sense, transport and coordinate group behavior by secreting one or several chemical molecules of small molecular weight. QS systems are widely distributed in bacteria, characterized by diversity and complexity. Acinetobacter baumannii biofilm is regulated by the QS system induced by N-acyl-homoserine lactones (AHLs) molecules. The exchange of information between bacteria through AHL molecules produced by a single bacterium causes a large accumulation of homologous bacteria. When the number of bacteria reaches a critical level, AHL becomes an effective sensing signal that prompts the production of a large number of intercellular adhesins in the polysaccharides, and the biofilm is formed by embedding the micro-colonies. ${ }^{[23]}$ However, Stacy et al. ${ }^{[24]}$ demonstrated that Acinetobacter baumannii used non-N- 
acylserine lactones in decaying population induction. Bacteria, through the QS system, could coordinate with each other in the same species as well as the number of other species to regulate their own behavior, and the interaction between different strains resulted in multiple infections. Liou et al. ${ }^{[25]}$ confirmed that the sensor kinase BfmS was also involved in biofilm formation. When the sensor kinase BfmS was absent, biofilm formation was significantly reduced, which provided a new theory for further research on biofilm control.

\subsection{Immune escape}

The formation of Acinetobacter baumannii biofilm can escape the body's immune function. The expression of complement transformation, neutrophil respiration and macrophage phagocytosis induced by biofilm significantly decreases. ${ }^{[26]}$ The Acinetobacter baumannii in biofilms can grow under the protection of alginate, and it is not easy to be removed. At the same time, the biofilm bacteria can stimulate the body to produce more antibodies, which makes it easier to form immune complexes with the corresponding soluble antigen to damage the surrounding body tissues, even aggravate the damage.

In short, the resistance of Acinetobacter baumannii biofilm is not single, but a result of many mechanisms though the theory has not yet been fully elucidated. With the further study of the related researches, the mechanism of the resistance to the biofilm will be veiled.

\section{Prevention and control of drug resis- tance mechanism of Acinetobacter bau- mannii biofilm}

Acinetobacter baumannii biofilm has drawn more and more attention as the detection rate of Acinetobacter baumannii increases as well as the rate of drug resistance. How to solve the biofilm resistance has become a hot research topic at present. Some scholars think that inhibition of bacterial signaling system and regulation of activated genes, ${ }^{[23]}$ prevention and treatment of biofilm formation are the most promising breakthroughs to eliminate Acinetobacter baumannii biofilms, and the biofilms must be destroyed in time.

\subsection{Inhibition of Acinetobacter baumannii biofilm formation}

The biofilm formation and maturation of Acinetobacter baumannii relies on the adhesion of microorganism to the surface of the carrier and the copolymerization between microorganisms, so inhibition of initial adhesion and aggregation of bacteria is more critical. Application of new ma- terials such as hydrophilic polymer materials and catheter surface coated with silver ions, chlorhexidine, ${ }^{[27]}$ titanium dioxide nanoparticles, hydrogel-coated ionic fluoroplastics, etc., can inhibit the value of Acinetobacter baumannii and prevente the formation of biofilms. ${ }^{[28]}$ The monoclonal antibody generated from the EPS of Acinetobacter baumannii can destroy EPS. It not only reduces the adhesion of bacteria but also increases the permeability of antibiotics to the biofilm. The growth of Acinetobacter baumannii is not affected no matter the QS system of Acinetobacter baumannii is weakened or destroyed. However, the communication between Acinetobacter baumannii and group QS is blocked, so that Acinetobacter baumannii can not be used as a group to regulate the formation of biofilm. It can effectively improve the killing and scavenging effects of antibiotics and immune system. Closantel, penicillin secondary metabolites (patulin and penicillic acid), halogenated furanone, methyl alkanoic acid salt, garlic extract, red algae and grapefruit juice can destroy the QS system through different mechanism. ${ }^{[28,29]}$ Studies have found that c-di-GMP is an important second messenger in the Acinetobacter baumannii signal transduction process. It plays an important role in the formation of biomembrane. Low concentration of exogenous c-di-GMP contributes to produce motility and toxicity factors, prevent bacterial adhesion and inhibit the formation of Acinetobacter baumannii biofilms, ${ }^{[30]}$ but high concentrations of c-di-GMP can promote the biofilm formation. ${ }^{[31]}$ The effect of c-di-GMP on Acinetobacter baumannii biofilm requires a deeper study. Gaseous nitric oxide (gNO) is shown to be effective in inhibiting Acinetobacter baumannii biofilm formation after $24 \mathrm{~h}$ of application. The effect of gNO release is time- and speed-dependent, which is better than other currently used antimicrobial agents. ${ }^{[32]}$ The antimicrobial peptide Kappacin, antimicrobial peptide LL-37 and fragment, ${ }^{[33]}$ biofilm degrading enzyme, serine protease $\mathrm{PKF}^{[34]}$ and quorum-quenching lactonase ${ }^{[35]}$ can eliminate Acinetobacter baumannii biofilm matrix and kill bacteria in the biofilm at the same time. In the case of acute infections, different forms of inhibition of Acinetobacter baumannii biofilms should be used, along with the rational use of antibiotics based on the drug's susceptibility to bacteria, ${ }^{[36]}$ which delays both drug resistance and chronic infection without turning them into chronic state and hinders its formation.

\subsection{Destruction of the formed biofilm of Acineto- bacter baumannii}

The mature biofilm is 10-1,000 times stronger than that of the planktonic bacteria. Formed biofilm of Acinetobacter baumannii is more difficult to obliterate, so how to destroy the biofilm and make it sensitive to antibiotics again is very important. Macrolides drugs have a damaging effect on the biofilm. A small dose of 14-, 15-membered ring macrolide antibiotics such as erythromycin, clarithromycin, 
roxithromycin, azithromycin, fosfomycin ${ }^{[37]}$ can destroy Acinetobacter baumannii biofilm structure and promote the penetration of sensitive antimicrobial agents. Therefore, the combination of sensitive antibiotics and small dose of macrolide can be more effective for the refractory infection of Acinetobacter baumannii biofilm. Ethanol, sodium chloride and soybean oil, cetyl chloride are able to destroy the Acinetobacter baumannii biofilm. ${ }^{[38]}$ Physical methods such as electric current, ultrasonic vibration, high-frequency pulse and heating can destroy the mature Acinetobacter baumannii biofilm to increase the antibiotic sensitivity to bacteria. Low frequency $(0.4 \mathrm{MHz})$ ultrasound can promote the penetration of oxygen molecules and nutrients in the biofilm and accelerate the release of bacterial metabolites, resulting in the recovery of low-metabolizing bacteria and the restoration of sensitivity to antibiotics. ${ }^{[39]}$ Chinese herbal medicine can also inhibit the formation of Acinetobacter baumannii biofilm. When combined with antibiotics, the antibacterial capacity of antibiotics is enhanced. In recent years, some achievements have been made in the single-flavored Chinese medicine, the compound preparation of Chinese traditional medicine, the monomer and its derivatives of the active ingredients of Chinese medicine. Flos lonicerae, Wuwei xiaodu pulvis, Shuanghuanglian, Chinese gallnut extract and ginseng monomer ${ }^{[40-42]}$ have the effect of inhibiting or destroying the biofilm. The bacteriophage AB7-IBB1, AB7-

\section{References}

[1] Wang LF, Li JL, Ma WH, et al. Drug resistance analysis of 1914 bacterial strains isolated from burn patients. Genet Mol Res. 2014.

[2] Ma MY, Xu J, Yu N, et al. Analysis of drug resistance of Acinetobacter baumannii and its related factors in ICU. Zhonghua Wei Zhong Bing Ji Jiu Yi Xue. 2013; 25(11): 686-689. PMid: 24225215.

[3] Chopra T, Marchaim D, Awali RA, et al. Epidemiology of bloodstream infections caused by Acinetobacter baumannii and impact of drug resistance to both carbapenems and ampicillinsulbactam on clinical outcomes. Antimicrob Agents Chemother. 2013; 57(12): 6270-6275. PMid: 24100492. https://doi.org/10.1128/AAC. 01520-13

[4] Gurung J, Khyriem AB, Banik A, et al. Association of biofilm production with multidrug resistance among clinical isolates of Acinetobacter baumannii and Pseudomonas aeruginosa from intensive care unit. Indian J Crit Care Med. 2013; 17(4): 214-218. PMid: 24133328. https://doi.org/10.4103/0972-5229.118416

[5] Archer NK, Mazaitis MJ, Costerton JW, et al. Staphylococcus aureus biofilms: properties, regulation, and rolesin human disease. Virulence. 2011; 2(5): 445-459. PMid: 21921685. https://doi. org/10.4161/viru.2.5.17724

[6] Wang LF. We should pay attention to the basic and clinical study of refractory wound injury and repair. Chinese Journal of Injury Repair and Wound Healing (Electronic version). 2012; 7(4): 4-6.

[7] Bhargava N, Sharma P, Capalash N. N-acyl homoserine lactone mediated interspecies interactions between $A$. baumannii and $P$. aeruginosa. Biofouling. 2012; 28(8): 813-822. PMid: 22867087. https://doi.org/10.1080/08927014.2012.714372

[8] Feng SH, Stojadinovic A, Izadjoo M, et al. Distinctive stages and strain variations of A. baumannii biofilm development under shear flow. J Wound Care. 2013; 22(4): 173-174, 176-178,
IBB2 and OligoG ${ }^{[27,43-45]}$ can promote dissociation and release of biofilm. Nanoparticles break the existing mechanism of resistance, including the reduction of drug absorption and the inhibition of the formation of Acinetobacter baumannii biofilm. ${ }^{[46]}$ Immune enhancer transfer factor, gamma globulin, thymidine, levamisole can improve the body's ability to erase the formation of BBF bacteria.

In summary, the status of Acinetobacter baumannii infection is not optimistic. It is a comprehensive and arduous task to prevent and treat Acinetobacter baumannii infection in biofilm formation. We should strengthen the concept of prevention as the mainstay in combination of prevention and treatment, and pay more attention to prevention and rational use of antibiotics. Rational use of antibiotics, combined with various methods to prevent and cure the biofilm formation of Acinetobacter baumannii, and remove the biofilm formed in time. Moreover, further study to explore the mechanisms of BBF resistance is required especially gene-oriented mechanisms, which can be more effective treatment of Acinetobacter baumannii caused by a variety of infections.

\section{Conflicts of Interest Disclosure}

The authors have no conflicts of interest related to this article.

180-181. PMid: 23702669. https://doi.org/10.12968/jowc . 2013.22 .4 .173

[9] Rumbo-Feal S, Gómez MJ, Gayoso C, et al. Whole transcriptome analysis of Acinetobacter baumannii assessed by RNA-sequencing reveals different mRNA expression profiles in biofilm compared to planktonic cells. PLoS One. 2013; 8(8): e72968. PMid: 24023660. https://doi.org/10.1371/journal.pone. 0072968

[10] Dong R, Guan C, Hu D, et al. The correlation study on antimicrobial resistance and biofilm related genes in clinical isolates of Acinetobacter baumannii. Zhonghua Wei Zhong Bing Ji Jiu Yi Xue. 2013; 25(8): 493-494. PMid: 24021047.

[11] Wei X, Shen D, Luo Y, et al. Molecular Mechanism of Biofilm Formation in Acinetobacter baumannii. Chinese Journal of Nosocomiology. 2010; 18(21): 2735-2738.

[12] Giannouli M, Antunes LC, Marchetti V, et al. Virulence-related traits of epidemic Acinetobacter baumannii strains belonging to the international clonal lineages I-III and to the emerging genotypes ST25 and ST78. BMC Infect Dis. 2013; 13: 282. PMid: 23786621. https://doi.org/10.1186/1471-2334-13-282

[13] Xiang J, Sun Z, Yang XG, et al. Changes in expression of gene abaI in biofilm of Acinetobacter baumannii strains isolated from burn patients. Zhonghua Shao Shang Za Zhi. 2012; 28(2): 101-105. PMid: 22781320.

[14] Zhang S, Zhang LP. Study on the clinical biofilm ability of Acinetobacter Bauman. Chinese Journal of Microbiology. 2011; 23(12): 1107-1109.

[15] Goh HM, Beatson SA, Totsika M. Molecular analysis of the Acinetobacter baumannii biofilm-associated protein. Appl Environ Microbiol. 2013; 79(21): 6535-6543. PMid: 23956398. https://doi. org/10.1128/AEM. 01402-13

[16] Jacobs AC, Blanchard CE, Catherman S. An Ribonuclease T2 Family Protein Modulates Acinetobacter baumannii. Abiotic Surface Colonization. 2014; 9(1): e85729. 
[17] Zhang XY, Ma DG. Study of the relationship between the membrane formation ability and genotyping of Acinetobacter baumannii. Beijing Medical Journal. 2011; 33(9): 728-730.

[18] Lewis K. Multidrug tolerance of biofilms and persister cells. Curr Top Microbiol Immunol. 2008; 322: 107-131. PMid: 18453274. https ://doi.org/10.1007/978-3-540-75418-3_6

[19] Lees-Miller RG, Iwashkiw JA, Scott NE. A common pathway for O-linked protein-glycosylation and synthesis of capsule in Acinetobacter baumannii. Mol Microbiol. 2013; 89(5): 816-830. PMid: 23782391. https://doi.org/10.1111/mmi. 12300

[20] Cuccui J, Wren BW. Bacteria like sharing their sweets. Mol Microbiol. 2013; 89(5): 811-815. PMid: 23844892. https://doi.org/ $10.1111 / \mathrm{mmi} .12328$

[21] Barth VC Jr, Rodrigues BÁ, Bonatto GD, et al. Heterogeneous persister cells formation in Acinetobacter baumannii. PLoS One. 2013; 8(12): e84361. PMid: 24391945. https://doi.org/10.1371/ journal.pone.0084361

[22] Djeribi R, Boucherit Z, Bouchloukh W, et al. A study of pH effects on the bacterial surface physicochemical properties of Acinetobacter baumannii. Colloids Surf B Biointerfaces. 2013; 102: 540-545. PMid: 23104023. https://doi.org/10.1016/j.colsurfb. 2012.08 .047

[23] Bitrian M, Solari CM, González RH, et al. Identification of virulence markers in clinically relevant strains of Acinetobacter genospecies. Int Microbiol. 2012; 15(2): 79-88. PMid: 22847269.

[24] Stacy DM, Welsh MA, Rather PN, et al. Blackwell HE. Attenuation of quorum sensing in the pathogen Acinetobacter baumannii using non-native N-Acyl homoserine lactones. ACS Chem Biol. 2012; 7(10): 1719-1728. PMid: 22853441. https://doi.org/ 10.1021/cb300351x

[25] Liou ML, Soo PC, Ling SR, et al. The sensor kinase BfmS mediates virulence in Acinetobacter baumannii. J Microbiol Immunol Infect. 2013.

[26] Thurlow LR, Hanke ML, Fritz T, et al. Staphylococcus aureus biofilms prevent macrophage phagocytosis and attenuate inflammation in vivo. Immunol. 2011; 186(11): 6585-6596. PMid: 21525381. https://doi.org/10.4049/jimmunol.1002794

[27] Jamal MA, Rosenblatt JS, Hachem RY. Prevention of biofilm colonization by gram-negative bacteria on minocycline-rifampinimpregnated catheters sequentially coated with chlorhexidine. Antimicrob Agents Chemother. 2014; 58(2): 1179-1182. PMid: 24165191. https://doi.org/10.1128/AAC.01959-13

[28] Wang LF, Li JL. To investigate the wound with bacteria. Chinese Journal of Injury Repair and Wound Healing. 2012; 7(4): 7-11.

[29] Nidadavolu P, Amor W, Tran PL, et al. Garlic ointment inhibits biofilm formation by bacterial pathogens from burn wounds. J Med Microbiol. 2012; 61(Pt 5): 662-671. PMid: 22301617. https : //doi.org/10.1099/jmm.0.038638-0

[30] Sambanthamoorthy K, Luo C, Pattabiraman N, et al. Identification of small molecules inhibiting diguanylate cyclases to control bacterial biofilm development. Biofouling. 2014; 30(1): 17 28. PMid: 24117391. https://doi.org/10.1080/08927014. 2013.832224

[31] Guan WJ, Wu MS, He CY. Pathogenic effect of c-di-GMP signal pathway on bacteria. Microbiology China. 2009; 36(3): 427-431.

[32] Sulemankhil I, Ganopolsky JG, Dieni CA, et al. Prevention and treatment of virulent bacterial biofilms with an enzymatic nitric oxide-releasing dressing. Antimicrob Agents Chemother. 2012;
56(12): 6095-6103. PMid: 22948868. https://doi.org/10. 1128/AAC. 01173-12

[33] Feng X, Sambanthamoorthy K, Palys T, et al. The human antimicrobial peptide LL-37 and its fragments possess both antimicrobial and antibiofilm activities against multidrug-resistant Acinetobacter baumannii. Peptides. 2013; 49: 131-137. PMid: 24071034. https://doi.org/10.1016/j.peptides.2013.09.007

[34] King LB, Pangburn MK, McDaniel LS, et al. Serine protease PKF of Acinetobacter baumannii results in serum resistance and suppression of biofilm formation. J Infect Dis. 2013; 207(7): 1128-1134. PMid: 23303803. https://doi.org/10.1093/infdis/jis939

[35] Chow JY, Yang Y, Tay SB, et al. Yew WS Disruption of Biofilm Formation by the Human Pathogen Acinetobacter baumannii using Engineered Quorum-quenching Lactonases. Antimicrob Agents Chemother. 2013

[36] Wang AL, Xue H. Clinical distribution of Acinetobacter baumannii and analysis of antimicrobial susceptibility. China Modern Medicine. 2011; 18(20): 80-81.

[37] Ozbek B, Mataraci E. In vitro effectiveness of colistin, tigecycline and levofloxacin alone and combined with clarithromycin and/or heparin as lock solutions against embedded Acinetobacter baumannii strains. J Antimicrob Chemother. 2013; 68(4): 827-830. PMid: 23203948. https://doi.org/10.1093/jac/dks472

[38] Hwang YY, Ramalingam K, Bienek DR, et al. Antimicrobial activity of nanoemulsion in combination with cetylpyridinium chloride in multidrug-resistant Acinetobacter baumannii. Antimicrob Agents Chemother. 2013; 57(8): 3568-3575. PMid: 23669390. https ://doi.org/10.1128/AAC .02109-12

[39] Karosi T, Sziklai I, Csomor P. Low-frequency ultrasound for biofilm disruption in chronic rhinosinusitis with nasal polyposis: in vitro pilot study. Laryngoscope. 2013; 123(1): 17-23. PMid: 22893599. https://doi.org/10.1002/lary. 23633

[40] He M. Study on the inhibitory effect of honeysuckle on bacterial biofilm and its chemical composition. Journal of Changchun University of Traditional Chinese. 2011.

[41] Wong RW, Hägg U, Samaranayake L, et al. Antimicrobial activity of Chinese medicine herbs against common bacteria in oral biofilm. A pilot study. Int J Oral Maxillofac Surg. 2010; 39(6): 599-605. PMid: 20418062. https://doi.org/10.1016/j.ijom.2010.02.024

[42] Zeng XP. Study on the inhibitory effect of AL-1 single use and combined use on the biofilm formation of Pseudomonas aeruginosa and its mechanism. Journal of Jinan University. 2011.

[43] Yele AB, Thawal ND, Sahu PK, et al. Chopade BA. Novel lytic bacteriophage AB7-IBB1 of Acinetobacter baumannii: isolation, characterization and its effect onbiofilm. Arch Virol. 2012; 157(8): 1441-1450. PMid: 22552486. https://doi.org/10. 1007/s00705-012-1320-0

[44] Thawal ND, Yele AB, Sahu PK, et al. Effect of a novel podophage AB7-IBB2 on Acinetobacter baumannii biofilm. Curr Microbiol. 2012; 65(1): 66-72. PMid: 22535475. https://doi.org/10. $1007 / \mathrm{s} 00284-012-0127-2$

[45] Powell LC, Sowedan A, Khan S, et al. The effect of alginate oligosaccharides on the mechanical properties of Gram-negative Biofilms. Biofouling. 2013; 29(4): 413-421. PMid: 23574333. https ://doi.org/10.1080/08927014.2013.777954

[46] Pelgrift RY, Friedman AJ. Nanotechnology as a therapeutic tool to combat microbial resistance. Adv Drug Deliv Rev. 2013; 65(13/14): 1803-1815. PMid: 23892192. https://doi.org/10.1016/j . addr.2013.07.011 